\title{
Infectious Complications in Pediatric Renal Transplant Patients
}

\author{
Orhan Deniz Kara ${ }^{\mathrm{a}}$, Nida Dincel, d, Ipek Kaplan Bulut ${ }^{\mathrm{a}}$, Kadriye Ozdemir ${ }^{\mathrm{a}}$, Ebru Yilmaz, \\ Zubeyir Hasan Gun ${ }^{\mathrm{b}}$, Cuneyt Hoscoskun ${ }^{\mathrm{c}}$, Betul Sozeria ${ }^{\mathrm{a}}$, Sevgi Mir ${ }^{\mathrm{a}}$
}

\begin{abstract}
Background: Infections in children with renal transplantation often lead to fatal complications. This study aimed to evaluate the incidence and risk factors of infection after renal transplantation, as well as its impact on graft function.
\end{abstract}

Methods: The study included 105 children, in two groups based on the presence of infection within the first 2 years after renal transplantation.

Results: In total, 137 infection episodes were found in $62(59 \%)$ patients. The number of episodes was 20, 5 and 54 that developed in months 6, 12 and 24 after transplantation, respectively. There were 72 urinary tract infection episodes. Among patients with pulmonary infections (16.7\%), two had H1N1, one of whom experienced graft loss and died of sepsis. The aspergillus infection episodes were lethal. Infection with BK virus was detected in four patients, all of whom were asymptomatic, except with decline in glomerular filtration rate. Seventeen patients developed 25 episodes of cytomegalovirus infection.

Conclusions: This study established the incidence of infections and risk factors in the first 2 years after transplantation at a single center. Long-term follow-up periods and prospective studies confirming specific risk factors, preventive measures and impact on graft survival are necessary.

Keywords: Renal transplantation; Infection; Children

Manuscript accepted for publication April 16, 2014

${ }^{a}$ Department of Pediatric Nephrology, Faculty of Medicine, Ege University, Izmir, Turkey

${ }^{b}$ Department of Pediatric Infectious Disease, School of Medicine, Emory

University, AT, USA

${ }^{\mathrm{c}}$ Department of General Surgery, Faculty of Medicine, Ege University, Izmir, Turkey

${ }^{\mathrm{d}}$ Corresponding author: Nida Dincel, Department of Pediatric

Nephrology, Faculty of Medicine, Ege University, Izmir, Turkey.

Email: nida_dincel@yahoo.com

doi: http://dx.doi.org/10.14740/wjnu159w

\section{Introduction}

In children with end-stage renal disease (ESRD), renal transplantation is the most important treatment of choice because of the better improvement of quality of life observed in patients compared to any dialysis method.

Infections in renal transplant patients are serious and often lead to fatal complications $[1,2]$. Outcome studies reporting on mortality rates and causes of graft loss in pediatric kidney recipients cite infections as the main cause.

Although the prevalence of infections varies from country to country, during the first year after renal transplantation, the prevalence was approximately $80 \%$ in the recipients $[2,3]$. Among renal transplant recipients, both children and adults, urinary tract infection (UTI) comprises the majority of infections, especially within the first year after kidney transplantation.

In the literature, there are very few reported studies about incidence and effect of infections in pediatric recipients. Also the risk factors of all infections after transplant have not been well-defined [4].

This study aimed to evaluate the incidence of all infections in renal transplant patients and to identify the risk factors associated with infection development, as well as its impact on graft function.

\section{Methods}

\section{Study population}

The study assessed 105 medical records of children who underwent renal transplantation in our center between January 1987 and May 2010. This is a retrospective study that established the incidence of infectious episodes in recipients in the first 2 years following transplantation. All patients were followed up for 2 years, or until death or graft loss if it occurred within the 2 years after transplantation.

Re-transplanted children were excluded from the study. The presence, number, types of infections and data on age, sex, donor type, ischemia time and primary causes of ESRD 
Table 1. Basic Characteristics of Patients

\begin{tabular}{ll} 
Patient characteristics & Patients $(\mathbf{n}=\mathbf{1 0 5})$ \\
\hline Recipient age (mean, years) & $7.9(1.5-18.1)$ \\
Male/female ratio & 0.98 \\
Body mass index & $18.5 \pm 1.3$ \\
Underlying disease of renal failure & \\
$\quad$ Nephrologic (n, \%) & $(52,49.5 \%)$ \\
$\quad$ Urinary (n, \%) & $(43,41 \%)$ \\
$\quad$ Unknown (n, \%) & $(10,9.5 \%)$ \\
RRT modality before tx: PD/HD/premptive (n) & $41 / 47 / 17$ \\
Cadaveric/living related donor (n) & $56 / 49$ \\
Mean ischemia time (h) & $15.3 \pm 11.1$ (ranges $2 \pm 25)$ \\
ICU stay (days) & $2.8 \pm 1.6$ \\
Central catheterization time (days) & $8.5 \pm 2.1$ \\
Urinary catheterization time (days) & $2.7 \pm 0.9$ \\
Double J use (patient number)/usage time (weeks) & $21 / 4.4 \pm 2$ weeks \\
Hospitalization time (days) & $19.8 \pm 5.2$ \\
\hline
\end{tabular}

RRT: renal replacement therapy; tx: transplantation; PD: peritoneal dialysis; HD: hemodialysis.

were analyzed. Patients were classified into two groups as an urologic or a nephrologic, according to the primary cause of ESRD. Additionally, all infectious episodes (type and time), graft function, episodes of acute rejection, immunosuppressive drug use, graft and patient survival, and duration of central and urethral catheterization were evaluated.

Site, type of infection and culture results were recorded in all patients. Peripheral blood and urine cultures as midstream catch were obtained in all children during febrile episodes $\left(>38{ }^{\circ} \mathrm{C}\right)$. In every infectious episode, glomerular filtration rate (GFR) was calculated, and proteinuria was measured from the 24-h collected urine.

\section{Infections}

The infectious events were identified by clinical signs and symptoms, as well as complementary laboratory assessment. Patients were divided into two groups based on the presence of infection.

UTI was defined as the presence of dysuria and pollakiuria, accompanied with fever and positive urine culture. UTIs were evaluated as upper UTIs according to findings of low urinary osmolality, high acute phase reactants and clinical clues including fever. Infections that occurred during ure- thral catheterization or within $24 \mathrm{~h}$ of catheter removal were considered catheter-related UTIs.

Respiratory infections were defined as the presence of respiratory insufficiency of any severity, accompanied by fever (temperature, $>38^{\circ} \mathrm{C}$ ), cough, dyspnea, purulent expectoration and radiologic exam revealing interstitial infiltrate $[3,5]$. Upper respiratory tract infections were diagnosed with $\geq 1$ of the following syndromes: common cold, pharyngitis, laryngitis, external otitis, otitis media, sinusitis, epiglottitis and acute bronchitis.

Epstein-Barr virus was detected based on the presence of viral capsid antigen IgG and viral capsid antigen IgM. The diagnosis of cytomegalovirus (CMV) disease was based on the presence of clinical symptoms (fever, malaise, arthralgia, myalgia, organ involvement with or without anemia and bicytopenia) and detection of CMV inclusion bodies in clinical samples (blood, urine, gastrointestinal tract mucosa and bronchoalveolar lavage fluid), and proven by anti-CMV antibody (IgG, IgM with ELISA method), CMV antigen and DNA PCR for CMV infection [1].

The diagnosis of herpetic infection was based only on clinical criteria: small vesicles and painful ulcers. Herpes zoster infection was identified with the presence of typical papulo-vesicular cutaneous eruptions or vesico-pustular le- 


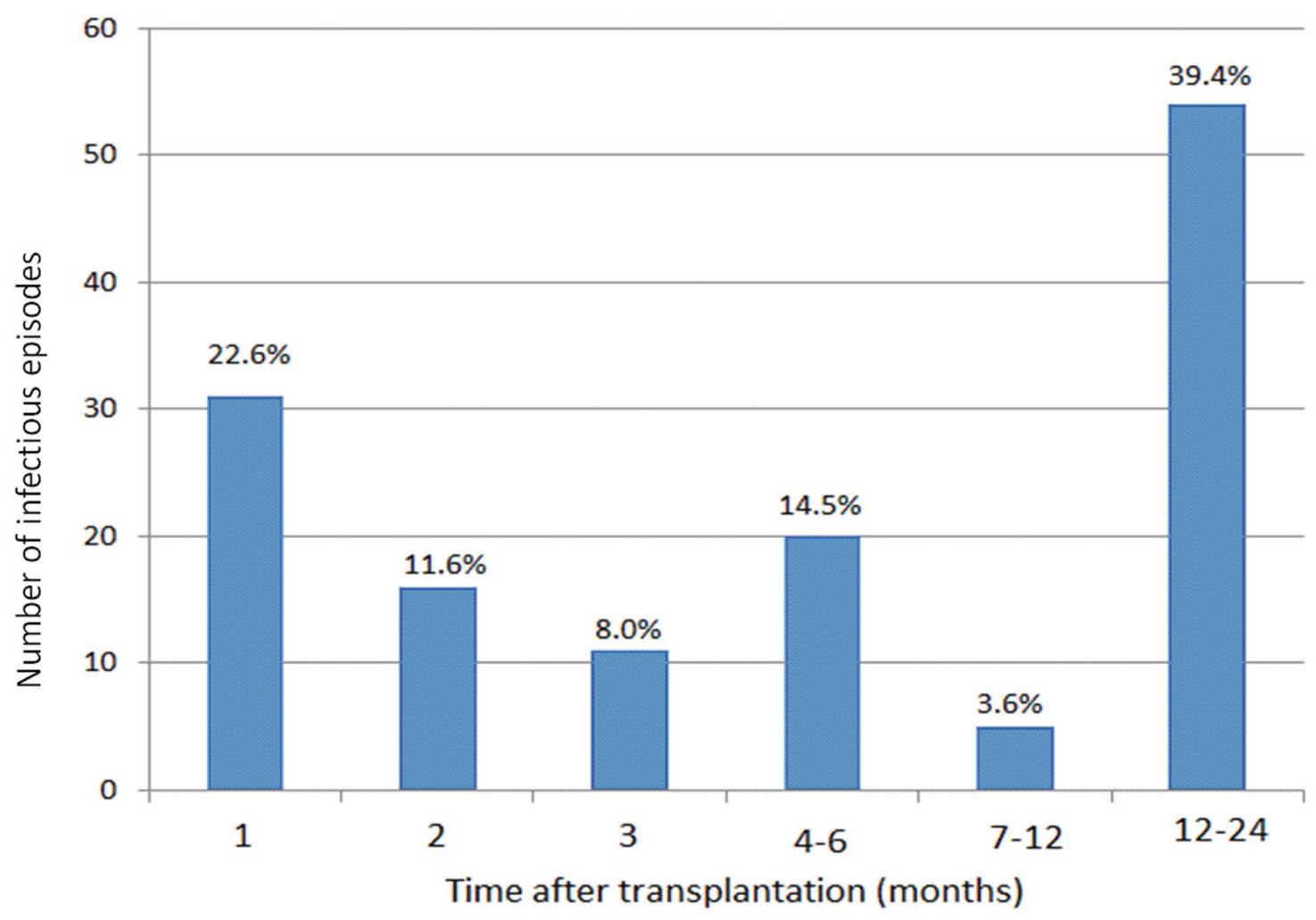

Figure 1. Number of episodes according to time after transplantation.

sions at specific dermatomes.

Bloodstream infection was proven with positive results of a peripheral blood culture, with or without fever or other clinical manifestations.

\section{Prophylactic regimen}

All patients were administered a preoperative and postoperative intravenous prophylactic antibiotic regimen. First generation cephalosporin was administered as a single dose during the preoperative period and for 4 days during the postoperative period. In addition, trimethoprim-sulfamethoxazole, acyclovir and nystatin were used as prophylaxis during the first 6 months after transplantation. No prophylactic agents were used for CMV, except in patients with CMV serology as donor positive and recipient negative.

\section{Immunosuppression}

Immunosuppression consisted of an induction therapy with basilixumab or anti-thymocyte globulin (ATG) (approximately 5 days) and $1 \mathrm{~g}$ prednisolone with a rapid decrease in week 1 after transplantation. Maintenance therapy consisted of corticosteroids, cyclosporine $\mathrm{A} /$ tacrolimus (calcineurin inhibitors) and mycophenolatemofetil/mycofenolate sodium. In older patients, corticosteroids, cyclosporine A and azathioprine were used in maintenance, but most of them were switched to mycophenolate. A majority were switched from calcineurin inhibitors to everolimus/sirolimus. ATG or a pulse of methylprednisolone was used to treat acute rejection.

\section{Statistical analysis}

The data were analyzed using the statistical software SPSS version 13.0 (SPSS, Chicago, IL, USA). Chi-square test was used for comparing categorical variables. For parametric data, the Mann-Whitney U test and the log-rank test were used as appropriate. The Kaplan-Meier method was used for univariate analyses of patient and graft survivals. Simple and multiple logistic regression analyses were performed to establish the contributions of the variables to the risk of infectious episodes. A P value $<0.05$ was considered statistically significant.

\section{Results}

\section{Patients}

The study group consisted of 105 pediatric (52 male, 53 female) renal transplant recipients with a median transplant 
Table 2. Association of Variables in Infected and Non-Infected Patients, Their Statistical Compare

\begin{tabular}{|c|c|c|c|}
\hline Variable & Infectious patients $(n=62)$ & Patients without infection $(n=43)$ & $\mathbf{P}$ \\
\hline Recipient age (years) & 11.3 & 13.0 & $<0.05$ \\
\hline Female & $36(58 \%)$ & $17(39.5 \%)$ & $<0.05$ \\
\hline Double J cathe (weeks) & $42.7 \pm 6.8$ & $42.7 \pm 4.9$ & 0.06 \\
\hline Hospitalization time (days) & $23 \pm 2.1$ & $19 \pm 3.2$ & 0.061 \\
\hline Urinary catheter duration (day) & $4.7 \pm 1.0$ & $4.5 \pm 1.03$ & 0.06 \\
\hline BMI & 17.05 & 18.6 & 0.08 \\
\hline Cadaveric kidney (n, \%) & $33(58.9 \%)$ & $23(41 \%)$ & $<0.05$ \\
\hline Haplotypes & $\begin{array}{l}\text { Identical,11.5\%; } \\
\text { haploidentical, } 30 \%\end{array}$ & $\begin{array}{l}\text { Identical, } 25.8 \% \text {; haploidentical, } \\
34.2 \%\end{array}$ & $<0.05$ \\
\hline Ischemia time (h) & $13.5 \pm 3.9$ & $15 \pm 4.3$ & 0.054 \\
\hline Donor age (years) & $34.8 \pm 13.5$ & $33.1 \pm 12$ & 0.08 \\
\hline $\begin{array}{l}\text { ATG induction during the first } \\
\text { week }\end{array}$ & $3.5 \pm 2.4$ & $4.5 \pm 2.9$ & $<0.05$ \\
\hline Dialysis time (months) & $19.8 \pm 18.9$ & $15.7 \pm 14.9$ & $<0.05$ \\
\hline
\end{tabular}

BMI: body mass index; ATG: anti-thymocyte globulin.

age of 7.9 years (range, 1.5 - 18.1 years). Table 1 shows the basic characteristics of all patients.

There were 43 patients in the urologic and 52 patients in the nephrologic groups. In 10 patients, the primary etiology was unknown. The most frequent underlying causes of renal failure were vesicoureteral reflux $(n=26)$, focal segmental glomerulosclerosis $(\mathrm{n}=20)$, chronic pyelonephritis $(\mathrm{n}=11)$ and posterior urethral valves $(n=9)$.

\section{Infectious episodes}

During 2 years, $59.0 \%$ of patients $(n=62)$ had at least 1 infectious episode (a total of 137 episodes) while $41.0 \%$ of patients had no infectious episode. Eighty-three (60.5\%) episodes were seen in the first year after transplantation. The incidence of infections per patient was 1.3 episodes/patient within the first year and 2.2 episodes/patients within the second year.

The incidence of infectious episodes was 31 (22.6\%), 16 $(11.6 \%)$ and $11(8 \%)$ in months 1, 2 and 3 after transplantation, respectively (Fig. 1). Episodes were more frequent in the first months after transplantation. The number of episodes was $20(14.5 \%), 5(3.6 \%)$ and $54(39.4 \%)$ in months 6,
12 and 24 after transplantation, respectively.

There were two deaths (3.2\%) during the 2 years among patients with infections. There were no deaths among patients with no infection $(\mathrm{P}<0.001)$.

While major signs and symptoms of infections were respiratory distress, fever, cough, purulent sputum, dysuria, polyuria, interrupted voiding, vesicular itchy and painful rash, some asymptomatic episodes were diagnosed with fluctuating renal function tests (especially serum creatinine levels) alone.

The following parameters significantly differed in patients with infectious episodes compared to those without infection: younger patients were more prone to infections than the older ones (11.3 vs. 13.0; $\mathrm{P}<0.05)$; predominance of females $(58.0 \%$ vs. $39.5 \% ; \mathrm{P}<0.05)$; longer dialysis time $(19.8 \pm 18.9$ months vs. $15.7 \pm 14.9$ months; $\mathrm{P}<0.05)$; lower human leukocyte antigen compatibility with the donor (identical, 11.5\%; haploidentical, $30.0 \%$ vs. identical, $25.8 \%$; haploidentical, $34.2 \% ; \mathrm{P}<0.05)$; increased number of grafts from deceased donors $(58.9 \%$ vs. $41.0 \%$; $\mathrm{P}<0.05)$; and lower ATG doses in the first induction therapy ( $3.5 \pm 2.4$ vs. 4.5 \pm 2.9 ; $\mathrm{P}<0.05$ ). No significant difference was observed in the cold ischemia time, prednisone use and other parameters 
Table 3. Bacterial Agents of Infections

Pathogens of UTI

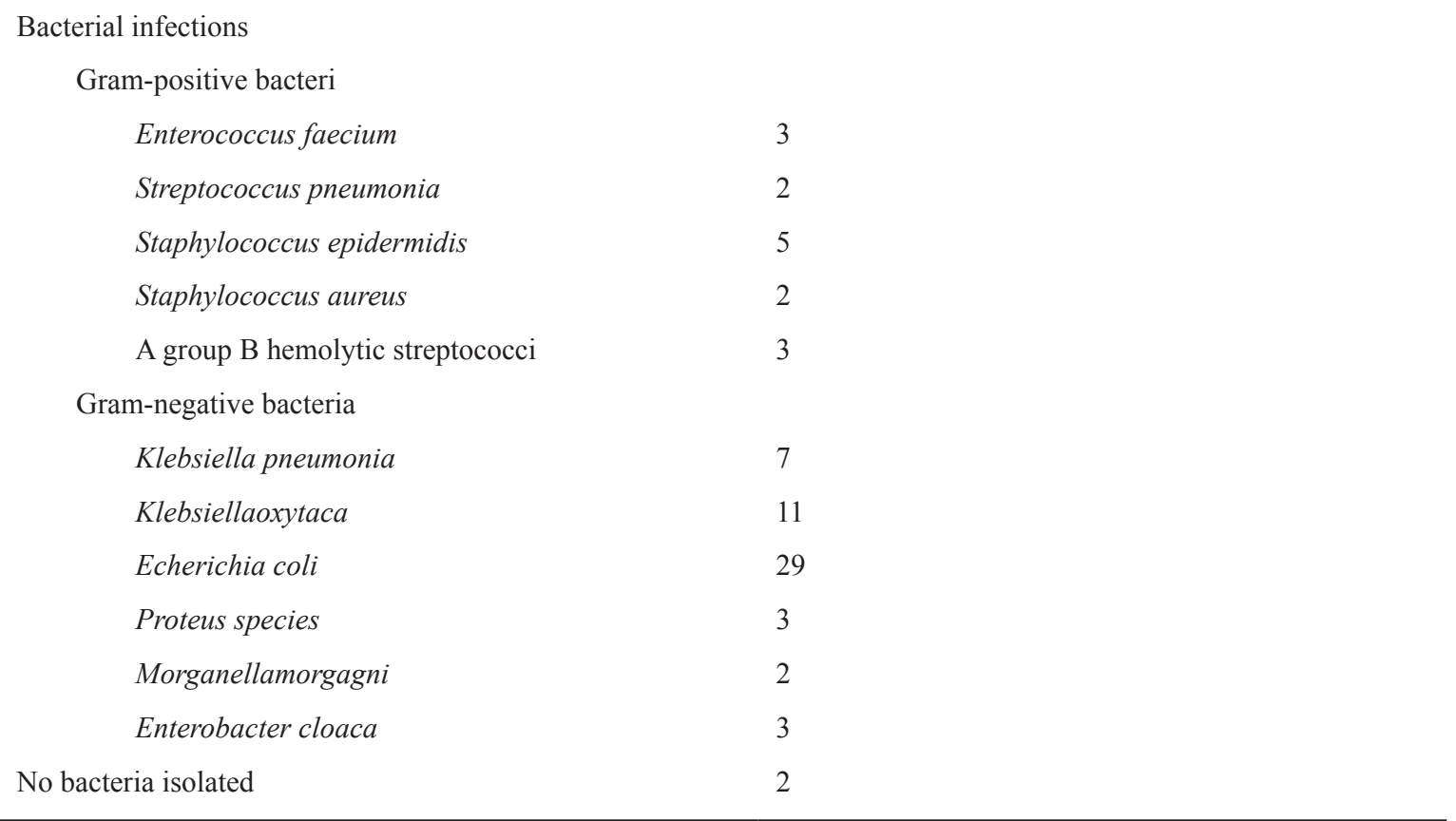

UTI: urinary tract infection.

shown in Table 2.

\section{Types of infections}

Viral infections were diagnosed mainly between months 2 and 6 after transplantation, while bacterial infections occurred throughout the follow-up period.

The mean age of the patients with UTI and without infection was $11.02 \pm 3.8$ and $12.7 \pm 3.7$ years, respectively. The lower age of patients with UTI was statistically significant $(\mathrm{P}=0.026)$.

There were 72 UTI episodes in 23 recipients (17 in the urologic, six in the nephrologic groups), of which 16 had only one UTI episode. Patients with recurrent infections $(\mathrm{n}=$ 7) had neurogenic bladder. Among the 72 episodes, 15 acute pyelonephritis (APN) episodes were noted; 13 of which occurred in patients with neurogenic bladder. The urologic group had $71 \%(\mathrm{n}=51)$ of the UTI episodes.

The incidence of UTI was higher in the urologic group than in the nephrologic group: $73.9 \%(\mathrm{n}=17)$ and $26 \%(\mathrm{n}=$ $6)$, respectively $(\mathrm{P}<0.0001)$.

Among the UTIs, gram-negative isolates were fourfold as frequent as gram-positive infections (76.3\% vs. $20.8 \%$ ), and no anerobic microorganisms were isolated. Escherichia coli was the most commonly isolated microorganism from the urine cultures.

Of the 72 episodes of UTI, the etiologic agent was $E$. coli in 29 (40\%), Klebsiellaoxytoca in $11(15.2 \%)$ and Klebsiellapneumoniae in $7(9.7 \%)$. The distribution of all etiological agents was listed in Table 3 . The urinary culture controls in patients with UTIs were negative on day 3 of treatment, except for $8(11.1 \%)$ episodes in only two patients with neurogenic bladder.

Of the UTIs occurring during the hospital stay (on 2.1 mean days) just after transplantation, 9.7\% urinary catheterassociated UTIs. Patients with postoperative UTIs had longer duration of urethral catheterization than those without infection (2.8 days vs. 2.3 days; $\mathrm{P}<0.05)$.

Among patients with pulmonary infections (16.7\%), two had H1N1, one of whom experienced graft loss and then died of sepsis. Another patient had prolonged pulmonary infection without any graft dysfunction. Ten of 23 pulmonary episodes were caused by bacteria. The other severe episode was an aspergillous infection that caused patient death.

In the four patients with BK virus infection, all were asymptomatic, and diagnosed by GFR decline. The only treat- 
ment approach was a reduction of immunosuppressive drug dosage. In three patients, the GFR decline persisted after BK viral infection was resolved.

The etiology of four episodes of bloodstream infection was attributed to coagulase-negative Staphylococcus in three patients and Staphylococcus aureus in one patient.

The four episodes of herpetic infections were localized in the thoracic region in two patients and at other locations in the remaining two patients.

Sixty-five recipients were CMV-seropositive and 40 recipients were seronegative. Seventeen (26\%) patients developed 25 episodes of CMV infection (seven were recipient negative [R-] and donor negative [D-], six were R+D- and four were $\mathrm{R}+\mathrm{D}+$ ), occurring after a mean of 92 days after transplantation. The clinical presentation of CMV infection was fluctuating creatinine levels. Increased CMV DNA copies were found in the first laboratory examination of 12 patients, while the other five were negative for DNA copies in the first laboratory evaluation, and who were then re-evaluated by biopsy. Inclusion bodies were detected in three of five biopsies. Histological findings indicated tubular atrophy and interstitial fibrosis with varying degrees. All patients were treated with intravenous ganciclovir until CMV DNA copies decreased to cut-off levels. Oral treatment was then continued for 6 weeks with valganciclovir.

All patients experienced normalization of laboratory values, although some biopsies revealed pathological changes.

\section{Survival analysis}

The graft functional rates at 6 and 12 months after transplantation were significantly lower among patients with infectious episodes than among those without infection $(94.3 \%$ and $97.2 \%$ vs. $98.1 \%$ and $99.1 \%$, respectively; $\mathrm{P}<0.05)$.

\section{Discussion}

Successful renal transplantation strictly depends on good infection control, which remains a serious threat [6]. The objective of this study was to determine the incidence and risk factors for infections and their effect on graft function in pediatric renal transplant recipients.

The incidence of infectious episodes varies significantly in different studies, but it is usually higher in the first month of transplantation. While viral and fungal opportunistic infections predominate during the first 6 months after transplantation, community acquired infections are common after 6 months following transplantation [7-9]. Our data indicated that approximately a quarter of the total number of episodes occurred in the first month. On the other hand, at the end of 6 months, this ratio was typical of $>50 \%$ of infections. This division was less obvious than that reported in other papers. Maraha et al reported that $>85 \%$ occurred in the first months and Regina et al reported that $>50 \%$ of the infectious events occurred within the first 3 months $[1,3]$. This high ratio during the first months after transplantation results from risk factors such as the long duration of hospital stay in the intensive care unit and catheterization time, incompatibility of patient/parents and the intensive induction therapy [10]. The number of episodes gradually decreased after 6 months (3.6\% during months $7-12$ ), and increased during the second year. Both nosocomial and opportunistic infection ratios were $39.4 \%$ in this period. These results were incompatible with those of other studies [11]. We believe that this higher ratio after the decrease in infectious episodes observed between 7 and 12 months arises mostly from patient care. After the first year, when there are more concerns for rejection prevention, patients and their caregivers relax if the graft is functioning well and tend to reduce the attention paid to hygiene, nutrition and other factors.

Approximately $80 \%$ of all renal transplant recipients experienced at least one episode of infection during the first year $[8,12]$. We retrospectively studied 105 patients who experienced $60.5 \%$ of infection episodes in the first year. On the other hand, Alagaden et al found that, of 127 renal transplant patients, $51 \%$ of recipients had infectious complications [13]. Regina et al also found 49\% of infectious episodes during the first year after transplantation [3]. This result indicates that the incidence of infectious episodes in some studies is less than that reported in our study. Various variables affect this outcome: patient age, donor age, ischemia times, dialysis duration and duration/type of induction therapy.

Among the conditions studied, the risk of infection was significantly associated with cadaveric donor, younger recipient age and less ATG induction doses [14]. Similarly, in our study, female recipients, younger recipient age, cadaveric donor, longer dialysis time, lower human leukocyte antigen compatibility, urological cause (especially neurogenic bladder) of ESRD and lower ATG doses were observed to be more prone to infections.

Chuang et al identified several risk factors for infection such as female sex, advanced age, history of vesicoureteral reflux, azathioprine use and cadaveric donor [15]. We did not find any association of risk of infection with induction or maintenance therapy, except for ATG usage. In their study, Dantas et al reported risk factors such as deceased donor, duration of urinary catheterization and length of hospitalization [16].

Urinary tract infection (UTI) is the most common infection in renal transplant recipients, with a prevalence of 35 $80 \%$, mainly in the first months after transplantation [8]. In a study in Brazil, the frequency of UTI found was $63 \%$, and in a Spanish study, it was $46.6 \%$ [17]. In this paper, the UTI frequency was $52.5 \%$ (72/137 episodes), and approximately $50 \%$ of them occurred during the first 6 months.

Frequency of UTIs depends on many factors such as 
age, female sex, kidney function, co-morbid factors, type and amount of immunosuppression, and catheterization and/ or the hospitalization time. Stamm et al reported that the risk of UTI is increased by approximately $5 \%$ with each day of bladder catheterization [18]. The earliest possible removal of the bladder catheter may lead to a drop in the UTI rates [19]. There were no statistically significant differences in urinary catheterization times between patients with and without infections in our study ( 4.7 days vs. 4.5 days). In addition, the catheterization time in our study was less than that in other studies. In 2010, Sorto et al studied 176 recipients and the mean catheter removal time was 6.5 and 5.2 days in patients with and without infections, respectively [20].

Main etiology of UTI is gram-negative bacilli, E. coli being the most frequently isolated [15-21]. In the current study, the most frequently observed etiologic agents were $E$. coli in $29(40 \%), K$. oxytoca in $11(15.2 \%)$ and K. pneumoniae in $7(9.7 \%)$ infections. Chuang et al evaluated the causative uropathogens in 213 cases and found that the most common ones were E. coli (29\%), Enterococcus (24\%), Staphylococcus $(12 \%)$ and Klebsiella (10\%) [15]. The underlying diagnosis had an impact in our cohort like in other studies [22].

In the patients with the primary diagnosis of vesicoureteral reflux, posterior urethral valves and/or neurogenic bladder, 51 UTI episodes $(71 \%)$ were noted.

In recent studies, the incidence and morbidity of APN after transplantation is not well studied in adults. The prevalence of UTI is $15-33 \%$ in pediatric studies [23-26]. Ulrike et al reported that APN was detected in $21 \%$ of patients in their study [22].

Several other studies showed decrement of renal function during UTI, affecting graft survival [23]. In correspondence with the literature, bacterial infectious were more common than viral infections, with a minor incidence of fungal infections across the study years. The incidence of systemic fungal infection varies with the type of organ transplanted, occurring in $2-14 \%$ of kidney transplant recipients [7]. On the other hand, 2010 annual transplant report of North American Pediatric Renal Trials and Collaborative Studies showed that the incidence of viral and bacterial infections was close to each other. The number of episodes in LRDs was significantly lower than that in cadaveric donors [27].

$\mathrm{CMV}$ is the most common opportunistic infection with an incidence of 30-52.4\%, mostly in the first 6 months after transplantation. In a study, the incidence of CMV infection was reported as $19.4 \%$ [28, 29]. Most of these infections lead to CMV reactivation because of the production of infectionrelated cytokines [10]. However, in the present study we did not find any correlation between severe infection and CMV reactivation. Our patients presented with decreased renal function. CMV reactivation was associated with acute rejection similar to that seen in the literature [1].

Respiratory tract infections corresponded to 16.7 of all infectious episodes. Sirlei et al found the ratio to be $8.9 \%$.
The ratio differences in various studies may be attributed to the choice of antibiotics or sociocultural features of the population. Most of the respiratory tract infections were caused by bacterial agents and all of them were resolved. Mortality was observed in one patient with H1N1 and another with septicaemia followed by aspergillus infection.

Infections are still associated with significant morbidity, with graft failure as well as patient death in the early post-transplant period [12]. Mortality from infections has decreased over the years, from $73 \%$ before 1976 to $20 \%$ during 1994 - $1996[9,30]$. The mortality rate resulting from infections was $0.02 \%$ in the follow-up period in our center, being close to the ratio of $0.3 \%$ in the United States.

In conclusion, this study established the incidence of infectious episodes and risk factors in the first 2 years after transplantation at a single center. By increasing our knowledge of the risk factors and their effect on survival of both graft and patients, we can increase early preventive measures and management experience.

\section{References}

1. Maraha B, Bonten $H$, van Hooff $H$, Fiolet $H$, Buiting $\mathrm{AG}$, Stobberingh EE. Infectious complications and antibiotic use in renal transplant recipients during a 1-year follow-up. Clin Microbiol Infect. 2001;7(11):619-625.

2. Pourmand G, Salem S, Mehrsai A, Taherimahmoudi M, Ebrahimi R, Pourmand MR. Infectious complications after kidney transplantation: a single-center experience. Transpl Infect Dis. 2007;9(4):302-309.

3. Sousa SR, Galante NZ, Barbosa DA, Pestana JO. [Incidence of infectious complications and their risk factors in the first year after renal transplantation]. J Bras Nefrol. 2010;32(1):75-82.

4. Palmer SM, Alexander BD, Sanders LL, Edwards LJ, Reller LB, Davis RD, Tapson VF. Significance of blood stream infection after lung transplantation: analysis in 176 consecutive patients. Transplantation. 2000;69(11):2360-2366.

5. Cisneros JM, Munoz P, Torre-Cisneros J, Gurgui M, Rodriguez-Hernandez MJ, Aguado JM, Echaniz A. Pneumonia after heart transplantation: a multi-institutional study. Spanish Transplantation Infection Study Group. Clin Infect Dis. 1998;27(2):324-331.

6. Veroux M, Giuffrida G, Corona D, Gagliano M, Scriffignano V, Vizcarra D, Tallarita T, et al. Infective complications in renal allograft recipients: epidemiology and outcome. Transplant Proc. 2008;40(6):1873-1876.

7. Patel R, Paya CV. Infections in solid-organ transplant recipients. Clin Microbiol Rev. 1997;10(1):86-124.

8. Kumar MS, Cridge P, Molavi A, Stephan R, Abouna GM. Infectious complications in the first 100 days after renal transplantation. Transplant Proc. 1995;27(5):2705- 
2706.

9. Snydman DR. Infection in solid organ transplantation. Transpl Infect Dis. 1999;1(1):21-28.

10. Tolkoff-Rubin NE, Rubin RH. Urinary tract infection in the immunocompromised host. Lessons from kidney transplantation and the AIDS epidemic. Infect Dis Clin North Am. 1997;11(3):707-717.

11. Martin-Pena A, Cordero E, Fijo J, Sanchez-Moreno A, Martin-Govantes J, Torrubia F, Cisneros J. Prospective study of infectious complications in a cohort of pediatric renal transplant recipients. Pediatr Transplant. 2009;13(4):457-463.

12. Rubin RH, Wolfson JS, Cosimi AB, Tolkoff-Rubin NE. Infection in the renal transplant recipient. Am J Med. 1981;70(2):405-411.

13. Alangaden GJ, Thyagarajan R, Gruber SA, Morawski K, Garnick J, El-Amm JM, West MS, et al. Infectious complications after kidney transplantation: current epidemiology and associated risk factors. Clin Transplant. 2006;20(4):401-409.

14. Perico N, Cattaneo D, Sayegh MH, Remuzzi G. Delayed graft function in kidney transplantation. Lancet. 2004;364(9447):1814-1827.

15. Chuang P, Parikh CR, Langone A. Urinary tract infections after renal transplantation: a retrospective review at two US transplant centers. Clin Transplant. 2005;19(2):230-235.

16. Dantas SR, Kuboyama RH, Mazzali M, Moretti ML. Nosocomial infections in renal transplant patients: risk factors and treatment implications associated with urinary tract and surgical site infections. J Hosp Infect. 2006;63(2):117-123.

17. Garcia-Prado ME, Cordero E, Cabello V, Pereira P, Torrubia FJ, Ruiz M, Cisneros JM. [Infectious complications in 159 consecutive kidney transplant recipients]. Enferm Infecc Microbiol Clin. 2009;27(1):22-27.

18. Stamm WE. Catheter-associated urinary tract infections: epidemiology, pathogenesis, and prevention. Am J Med. 1991;91(3B):65S-71S.

19. Rabkin DG, Stifelman MD, Birkhoff J, Richardson KA, Cohen D, Nowygrod R, Benvenisty AI, et al. Early catheter removal decreases incidence of urinary tract infections in renal transplant recipients. Transplant Proc. 1998;30(8):4314-4316.
20. Sorto R, Irizar SS, Delgadillo G, Alberu J, Correa-Rotter $\mathrm{R}$, Morales-Buenrostro LE. Risk factors for urinary tract infections during the first year after kidney transplantation. Transplant Proc. 2010;42(1):280-281.

21. Sharifian M, Rees L, Trompeter RS. High incidence of bacteriuria following renal transplantation in children. Nephrol Dial Transplant. 1998;13(2):432-435.

22. John U, Everding AS, Kuwertz-Broking E, Bulla M, Muller-Wiefel DE, Misselwitz J, Kemper MJ. High prevalence of febrile urinary tract infections after paediatric renal transplantation. Nephrol Dial Transplant. 2006;21(11):3269-3274.

23. Dunn SP, Vinocur CD, Hanevold C, Wagner CW, Weintraub WH. Pyelonephritis following pediatric renal transplant: increased incidence with vesicoureteral reflux. J Pediatr Surg. 1987;22(12):1095-1099.

24. Mueller T, Resinger C, Ruffingshofer D, Arbeiter K, Balzar E, Aufricht C. Urinary tract infections beyond the early post-transplant period in pediatric renal graft recipients. Wien Klin Wochenschr. 2003;115(11):385388.

25. Ranchin B, Chapuis F, Dawhara M, Canterino I, HadjAissa A, Said MH, Parchoux B, et al. Vesicoureteral reflux after kidney transplantation in children. Nephrol Dial Transplant. 2000;15(11):1852-1858.

26. Neuhaus TJ, Schwobel M, Schlumpf R, Offner G, Leumann E, Willi U. Pyelonephritis and vesicoureteral reflux after renal transplantation in young children. J Urol. 1997;157(4):1400-1403.

27. North American Pediatric Renal Trials and Collaborative Studies 2010; Annual Transplant Report.

28. Breinig MK, Zitelli B, Starzl TE, Ho M. Epstein-Barr virus, cytomegalovirus, and other viral infections in children after liver transplantation. J Infect Dis. 1987;156(2):273-279.

29. Melgosa Hijosa M, Garcia Meseguer C, Pena Garcia P, Alonso Melgar A, Espinosa Roman L, Pena Carrion A, Navarro Torres M. Preemptive treatment with oral ganciclovir for pediatric renal transplantation. Clin Nephrol. 2004;61(4):246-252.

30. Splendiani G, Cipriani S, Tisone G, Iorio B, Condo $\mathrm{S}$, Vega A, Dominijanni S, et al. Infectious complications in renal transplant recipients. Transplant Proc. 2005;37(6):2497-2499. 